\title{
EFFECT OF ANTHOCYANIDINS ON TRASCRIPTIONAL ACTIVITIES OF STEROID AND NUCLEAR RECEPTORS
}

Barbora Pastorkova, Petr Illes, Zdenek Dvorak

Department of Cell Biology and Genetics, Faculty of Science, Palacky University, Slechtitelu 27, 78371 Olomouc, Czech Republic E-mail: barbor.pastorkova@gmail.com

\section{Introduction}

Anthocyanidins are aglycons of anthocyanins, which are plant pigments responsible for floral and fruits color. Anthocyanidins possess beneficial pharmacological properties such as antioxidant, anti-inflammatory, anti-tumor and anti-diabetic. Anthocyanidins are contained in normal diet and might play role in food-drug interactions [1]. Food-drug interactions proceed either by inhibition or induction of drug metabolizing enzymes. These enzymes are transcriptionally regulated by steroid and nuclear receptors [2]
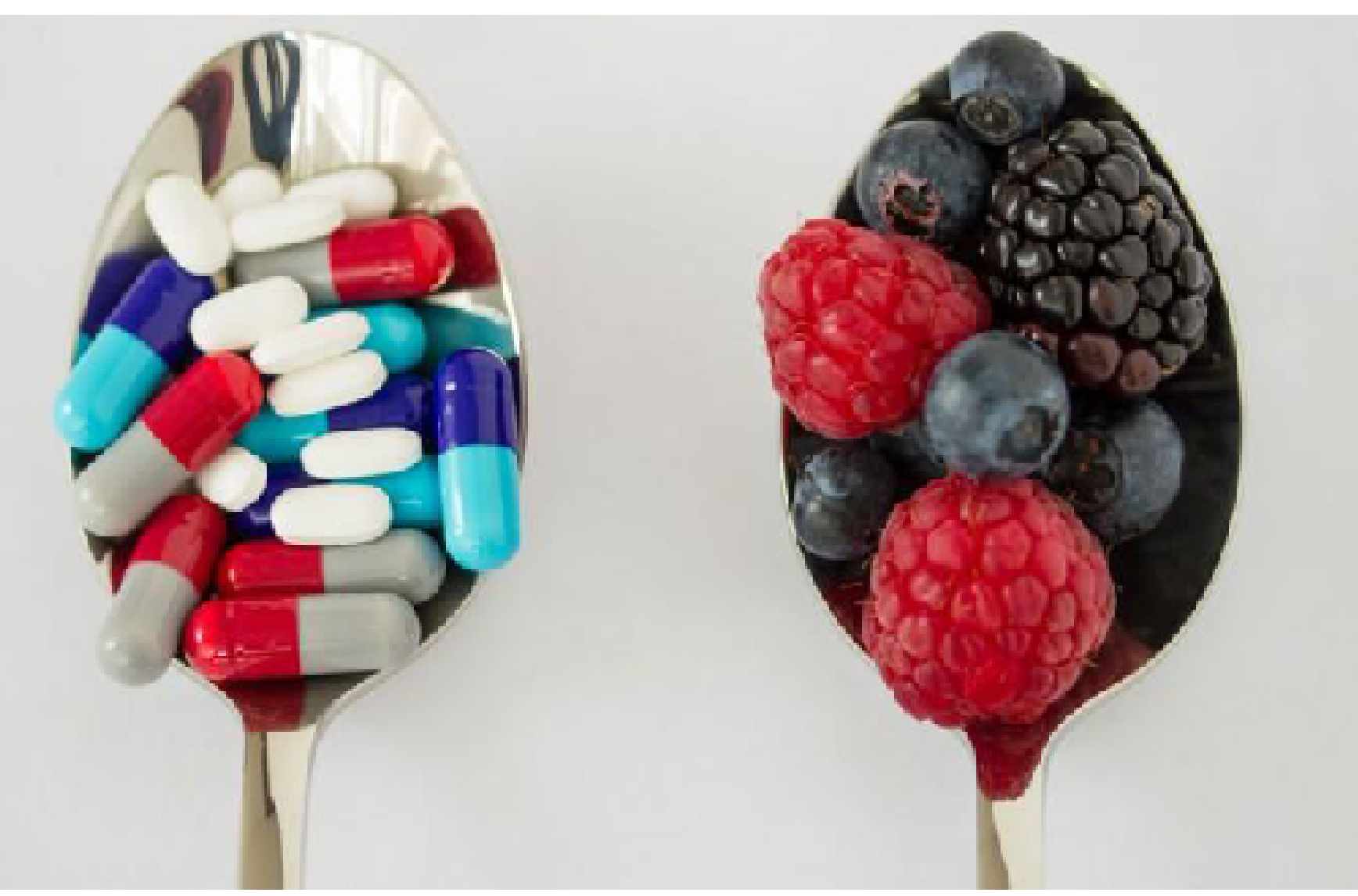

The aim of current study was to evaluate the effects of cyanidin, delphinidin malvidin, pelargonidin and peonidin on the transcriptional activities of steroid and nuclear receptors.
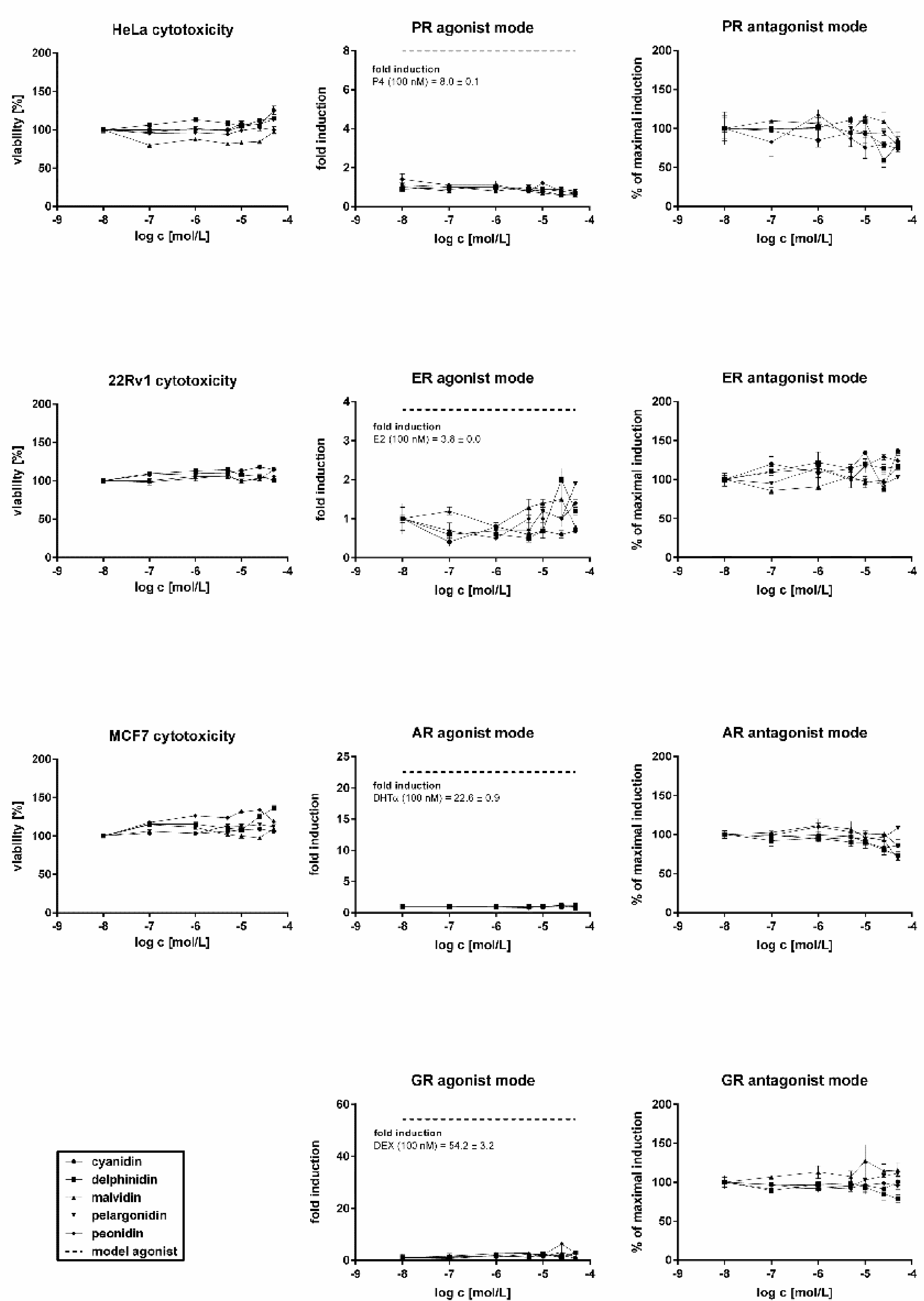

Fig. 1. Effect of anthocyanidins on transcriptional activity of steroid receptors.

The line charts show cytotoxicity of anthocyanidins on HeLa, MCF-7 and 22Rv1 cells (left column), luciferase activity of cells transfected with reporter plasmids for progesterone (PR), estrogen (ER), androgen (AR) and glucocorticoid receptors (GR) in agonist (middle column) and antagonist (right column) mode. The data represent the mean + SD of triplicate measurements and are expressed either as a percentage of viability (cytotoxicity), or as a fold induction over DMSO-treated cells (agonist mode), or as a percentage of the fold induction by model agonists (antagonist mode)

\section{Conclusion}

None of the tested anthocyanidins in given concentrations was cytotoxic in the cell lines or caused significant induction of transcriptional activity. We found that some anthocyanidins in $50 \mu \mathrm{M}$ concentration inhibited transcriptional activity of examined receptors. The inhibition down-to $49.4 \%$ for malvidin vs VDR, $49.3 \%$ for delphinidin vs RXR, $38.0 \%$ for malvidin vs RAR and $52.2 \%$ for pelargonidin vs PXR of maximal induction was observed.

\section{Methods}

The activities of glucocorticoid receptor (GR), androgen receptor (AR), estrogen receptor (ER), progesterone receptor (PR), thyroid receptor (TR), vitamin $D$ receptor $(V D R)$, pregnane $X$ receptor $(P X R)$, retinoic acid receptor (RAR) and retinoid $X$ receptor (RXR) were assessed using either stably or transiently transfected luciferase gene reporter cell lines. HeLa - GR, 22Rv1 - AR, PR, MCF7 - ER, HepG2 - TR, LS180 VDR, PXR, Hek293 - RXR, RAR were used. In case of transient transfection, reporter plasmids was transfected to the cell lines by lipofection (FuGENE ${ }^{\circledR}$ HD Transfection Reagent). The cytotoxicity assays and gene reporter assays were performed after the $24-h$ treatment of cells with increasing range of concentrations $(10 \mathrm{nM}-50 \mu \mathrm{M})$ of tested anthocyanidins. The experiments of gene reporter assay were performed in the absence (agonist mode) or in the presence (antagonist mode) of model agonist. Model agonists: dexamethasone (DEX), $5 \alpha$-dihydrotestosterone (DHT), 17 $\beta$-estradiol (E2), progesterone (P4), 3,3,5-triiodo-L-thyronine (T3), 1,25-dihydroxy vitamin D3 (VD3), rifampicin (RIF), all-trans-retinoic acid and (transRA) 9-cis-retinoic acid (cisRA) were used for GR, AR, ER, PR, TR, VDR, PXR, RAR and RXR, respectively. After the treatment, cells were lysed and luciferase activity was measured. The experiments were carried out in triplicates and in several independent cell passages.
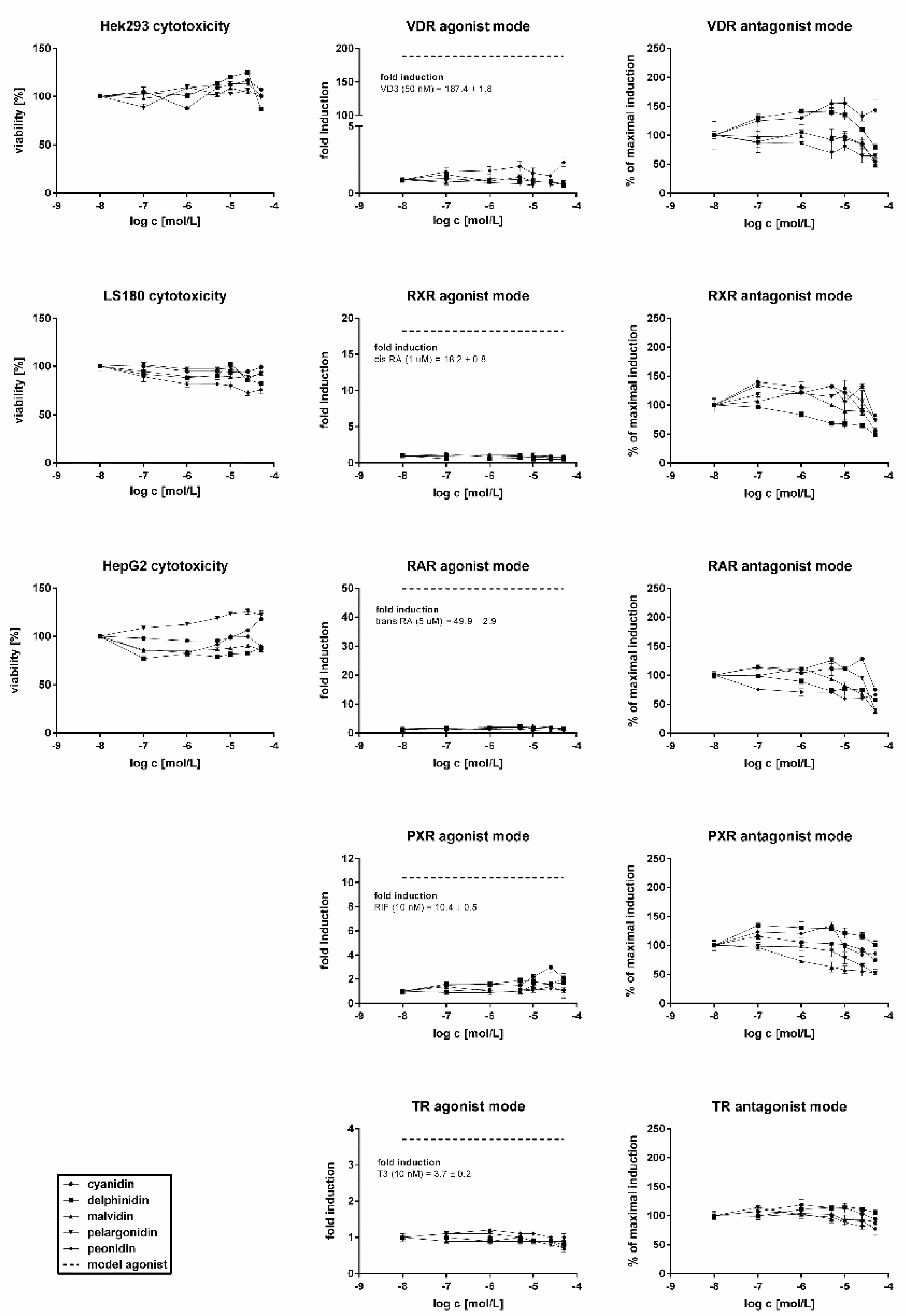

Fig. 2. Effect of anthocyanidins on transcriptional activity of nuclear receptors.

The line charts show cytotoxicity of anthocyanidins on HEK293, LS180 and HepG2 cells (left column), luciferase activity of cells transfected with reporter plasmids for vitamin $D$ (VDR), retinoid $X(R X R)$, retinoic acid (RAR), pregnane $X(P X R)$ and thyroid receptors (TR) in agonist (middle column) and antagonist (right column) mode. The data represent the mean \pm SD of triplicate measurements and are expressed either as a percentage of viability (cytotoxicity), or as a fold induction over DMSO-treated cells (agonist mode), or as a percentage of the fold induction by model agonists (antagonist mode)

\section{References}

[1] Kong JM, Chia LS, Goh NK, Chia TF, Brouillard R. Analysis and biological activities of anthocyanins. Phytochemistry. 2003; 64(5):923-33.

[2] Lin JH, Lu AY. Inhibition and induction of cytochrome P450 and the clinical implications. Clin Pharmacokinet. 1998; 35(5):361-90.

This work was supported by Czech Science Foundation P303/12/G163 